\title{
Monodromy Zeta-functions of Deformations and Newton Diagrams
}

\author{
Gleb GUSEV \\ Moscow State University \\ Independent University of Moscow \\ Moscow - Russia \\ gusev@mccme.ru
}

Received: May 29, 2008

Accepted: October 7, 2008

\begin{abstract}
For a one-parameter deformation of an analytic complex function germ of several variables, there is defined its monodromy zeta-function. We give a Varchenko type formula for this zeta-function if the deformation is non-degenerate with respect to its Newton diagram.
\end{abstract}

Key words: Deformations of singularities, monodromy, zeta-function, Newton diagram. 2000 Mathematics Subject Classification: 14B07, 32S30, 14D05, 58K10, 58K60.

\section{Introduction}

Let $F$ be the germ of an analytic function on $\left(\mathbb{C}^{n+1}, 0\right)$, where $\mathbb{C}^{n+1}=\mathbb{C}_{\sigma} \times \mathbb{C}_{\mathbf{z}}^{n}, \sigma$ is the coordinate on $\mathbb{C}$, and $\mathbf{z}=\left(z_{1}, z_{2}, \ldots, z_{n}\right)$ are the coordinates on $\mathbb{C}^{n}$. The germ $F$ provides a deformation $f_{\sigma}=F(\sigma, \cdot)$ of the function germ $f=f_{0}$ on $\left(\mathbb{C}^{n}, 0\right)$. We give formulae for the monodromy zeta-functions of the deformations of the hypersurface germs $\{f=0\} \cap\left(\mathbb{C}^{*}\right)^{n}$ and $\{f=0\}$ at the origin in terms of the Newton diagram of $F$. A reason to study deformations of hypersurface germs and their monodromy zeta-functions was inspired by their connection with zeta-functions of deformations of polynomials: [3].

Let $A$ be the complement to an arbitrary analytic hypersurface $Y$ in $\mathbb{C}^{n}: A=$ $\mathbb{C}^{n} \backslash Y$. Let $V=\{F=0\} \cap\left(\mathbb{C}_{\sigma} \times A\right) \cap B_{\varepsilon}$, where $B_{\varepsilon} \subset \mathbb{C}^{n+1}$ is the closed ball of

Partially supported by the grants RFBR-007-00593, INTAS-05-7805, NWO-RFBR 047.011.2004.026, RFBR-08-01-00110-a 
radius $\varepsilon$ with the centre at the origin. Let $\mathbb{D}_{\delta}^{*} \subset \mathbb{C}_{\sigma}$ be the punctured disk of radius $\delta$ with the centre at the origin. For $0<\delta \ll \varepsilon$ small enough the restriction to $V$ of the projection $\mathbb{C}^{n+1} \rightarrow \mathbb{C}_{\sigma}$ onto the first factor provides a fibration over $\mathbb{D}_{\delta}^{*}([7])$. Denote by $V_{c}$ the fibre over the point $c$. Consider the monodromy transformation $h_{F, A}: V_{c} \rightarrow V_{c}$ of the above fibration restricted to the loop $c \cdot \exp (2 \pi i t), t \in[0,1],|c|$ is small enough.

The zeta-function of an arbitrary transformation $h: X \rightarrow X$ of a topological space $X$ is the rational function $\zeta_{h}(t)=\prod_{i \geq 0}\left(\operatorname{det}\left(\operatorname{Id}-\left.t h_{*}\right|_{H_{i}^{c}(X ; \mathbb{C})}\right)\right)^{(-1)^{i}}$, where $H_{i}^{c}(X ; \mathbb{C})$ is the $i$-th homology group with closed support.

Definition 1.1. The zeta-function of the monodromy transformation $h_{F, A}$ will be called the monodromy zeta-function of the deformation $f_{\sigma}$ on $A: \zeta_{\left.f_{\sigma}\right|_{A}}(t)=\zeta_{h_{F, A}}(t)$.

For a power series $S=\sum c_{\mathbf{k}} \mathbf{y}^{\mathbf{k}}, \mathbf{y}^{\mathbf{k}}=y_{1}^{k_{1}} \cdots y_{m}^{k_{m}}$, one defines its Newton diagram as follows. Denote by $\mathbb{R}_{+} \subset \mathbb{R}$ the set of non-negative real numbers. Denote by $\Gamma^{\prime}(S)$ the convex hull of the union $\cup_{c_{\mathbf{k}} \neq 0}\left(\mathbf{k}+\mathbb{R}_{+}^{m}\right)$. The Newton diagram of the series $S$ is the union of compact faces of $\Gamma^{\prime}(S)$. For a germ $G$ on $\mathbb{C}^{m}$ at the origin, its Newton diagram $\Gamma(G)$ is the Newton diagram of its Taylor series at the origin.

For a generic germ $F$ on $\left(\mathbb{C}^{n+1}, 0\right)$ with fixed Newton diagram $\Gamma \in \mathbb{R}_{+}^{n+1}$ the zeta-functions $\zeta_{\left.f_{\sigma}\right|_{\left.\mathbb{C}^{*}\right)^{n}}}(t), \zeta_{\left.f_{\sigma}\right|_{\mathbb{C}^{n}}}(t)$ are also fixed. We provide explicit formulas for these zeta-functions in terms of the Newton diagram $\Gamma$.

\section{The main result (a Varchenko type formula)}

Let $F$ be a germ of a function on $\left(\mathbb{C}^{n+1}, 0\right)$. Let $\mathbf{k}=\left(k_{0}, k_{1}, \ldots, k_{n}\right)$ be the coordinates on $\mathbb{R}^{n+1}$ corresponding to the variables $\sigma, z_{1}, \ldots, z_{n}$ respectively. For $I \subset\{0,1, \ldots, n\}$, denote by $\mathbb{R}^{I}$ and $\Gamma^{I}(F)$ the sets $\left\{\mathbf{k} \mid k_{i}=0, i \notin I\right\} \subset \mathbb{R}^{n+1}$ and $\Gamma(F) \cap \mathbb{R}^{I}$ respectively.

An integer covector is called primitive if it is not a multiple of another integer covector. Let $P^{I}$ be the set of primitive integer covectors in the dual space $\left(\mathbb{R}^{I}\right)^{*}$ such that all their components are strictly positive. For $\alpha \in P^{I}$, let $\Gamma_{\alpha}^{I}(F)$ be the subset of the diagram $\Gamma^{I}(F)$ where $\left.\alpha\right|_{\Gamma^{I}(F)}$ reaches its minimal value: $\Gamma_{\alpha}^{I}(F)=\{\mathbf{x} \in$ $\left.\Gamma^{I}(F) \mid \alpha(\mathbf{x})=\min \left(\left.\alpha\right|_{\Gamma^{I}(F)}\right)\right\}$ (for $\Gamma^{I}(F)=\emptyset$ we assume $\Gamma_{\alpha}^{I}(F)=\emptyset$ ). Consider the Taylor series of the germ $F$ at the origin: $F=\sum F_{\mathbf{k}} \sigma^{k_{0}} z_{1}^{k_{1}} \ldots z_{n}^{k_{n}}$. Denote: $F_{\alpha}=\sum_{\mathbf{k} \in \Gamma_{\alpha}^{\{0,1, \ldots, n\}}} F_{\mathbf{k}} \sigma^{k_{0}} z_{1}^{k_{1}} \ldots z_{n}^{k_{n}}$.

Definition 2.1. A germ $F$ of a function on $\left(\mathbb{C}^{n+1}, 0\right)$ is called non-degenerate with respect to its Newton diagram if for any $\alpha \in P^{I}$ the 1 -form $d F_{\alpha}$ does not vanish on the germ $\left\{F_{\alpha}=0\right\} \cap\left(\mathbb{C}^{*}\right)^{n+1}$ at the origin (see $[9]$ ).

For $I \in\{0,1, \ldots, n\}$ such that $0 \in I$, we denote:

$$
\zeta_{F}^{I}(t)=\prod_{\alpha \in P^{I}}\left(1-t^{\alpha\left(\frac{\partial}{\partial k_{0}}\right)}\right)^{(-1)^{l-1} l ! V_{l}\left(\Gamma_{\alpha}^{I}(F)\right)}
$$


where $l=|I|-1, \frac{\partial}{\partial k_{0}}$ is the vector in $\mathbb{R}^{I}$ with the single non-zero coordinate $k_{0}=1$, and $V_{l}(\cdot)$ denotes the $l$-dimensional integer volume, i.e., the volume in a rational $l$ dimensional affine hyperplane of $\mathbb{R}^{I}$ normalized in such a way that the volume of the minimal parallelepiped with integer vertices is equal to 1 . We assume that $V_{0}(\mathrm{pt})=1$ and for $n \geq 0$ one has $V_{n}(\emptyset)=0$.

Theorem 2.2. Let $F$ be non-degenerate with respect to its Newton diagram $\Gamma(F)$. Then one has

$$
\begin{aligned}
\zeta_{\left.f_{\sigma} \mid \mathbb{C}^{*}\right)^{n}}(t) & =\zeta_{F}^{\{0,1 \ldots, n\}}(t), \\
\zeta_{f_{\sigma} \mid \mathbb{C}^{n}}(t) & =(1-t) \times \prod_{I: 0 \in I \subset\{0,1, \ldots, n\}} \zeta_{F}^{I}(t) .
\end{aligned}
$$

Remarks 2.3 .

(i) The equation (1) implies the equation (2) because of the following multiplicative property of the zeta-function. Let $h: X \rightarrow X$ be a transformation of a CW-complex $X$. Let $Y \subset X$ be a subcomplex of $X$. Assume that $h(Y) \subset Y$, $h(X \backslash Y) \subset(X \backslash Y)$. Then $\zeta_{\left.h\right|_{X}}(t)=\zeta_{\left.h\right|_{X \backslash Y}}(t) \times \zeta_{\left.h\right|_{Y}}(t)$.

One can see that $\zeta_{\left.f_{\sigma}\right|_{\{0\}}}(t)=(1-t) \times \zeta_{F}^{\{0\}}(t)$. In fact, in the case $\Gamma^{\{0\}}=\emptyset$ one has $\zeta_{\left.f_{\sigma}\right|_{\{0\}}}(t)=(1-t), \zeta_{F}^{\{0\}}(t)=1$. Otherwise $\zeta_{\left.f_{\sigma}\right|_{\{0\}}}(t)=1, \quad \zeta_{F}^{\{0\}}(t)=(1-t)^{-1}$.

(ii) The zeta-function $\zeta_{\left.f_{\sigma}\right|_{\mathbb{C} n}}(t)$ coincides with the monodromy zeta-function of the germ of the function $\sigma:\{F=0\} \rightarrow \mathbb{C}_{\sigma}$ at the origin. The main theorem of [8] provides a formula for the zeta-functions of germs of functions on complete intersections in nondegenerate cases. One can apply this formula to the germ $\sigma$ and verify that the formula (2) agrees with the one of M. Oka. But (2) can not be deduced from the result of M. Oka because the function $\sigma$ does not satisfy the condition of "convenience" ([8, page 17]).

Example 2.4.

(i) Let $F(\sigma, \mathbf{z})=f(\mathbf{z})-\sigma$. The monodromy zeta-function of the deformation $f_{\sigma}$ coincides with the (ordinary) monodromy zeta-function $\zeta_{f}(t)$ of the germ $f$ on $\left(\mathbb{C}^{n}, 0\right)$ (see, e.g., [9]). In this case the $l$-dimensional faces $\Gamma_{\alpha}^{I}(F)$ (where $l=|I|-1>0$ ) are cones of integer height 1 over the corresponding $(l-1)$-dimensional faces $\Gamma_{\left.\alpha\right|_{\left\{k_{0}=0\right\}}}^{I \backslash\{0\}}(f)$. One has:

$$
V_{l}\left(\Gamma_{\alpha}^{I}(F)\right)=V_{l-1}\left(\Gamma_{\left.\alpha\right|_{\left\{k_{0}=0\right\}}}^{I \backslash\{0\}}(f)\right) / l,
$$

with $\alpha\left(\partial / \partial k_{0}\right)=\min \left(\left.\alpha\right|_{\Gamma^{I \backslash\{0\}}(f)}\right)$. This means that in this case the equation (2) coincides with the Varchenko formula $([9])$.

(ii) For a deformation $F(\sigma, \mathbf{z})$ of the form $f_{0}(\mathbf{z})-\sigma f_{1}(\mathbf{z})$, the fibre

$$
\left(\{\sigma\} \times\left\{f_{\sigma}=0\right\}\right) \cap B_{\varepsilon}
$$

is the disjoint union of the sets

$$
\left(\{\sigma\} \times\left\{f_{0} / f_{1}=\sigma\right\}\right) \cap B_{\varepsilon}
$$


and

$$
\left(\{\sigma\} \times\left\{f_{0}=f_{1}=0\right\}\right) \cap B_{\varepsilon} .
$$

If $f_{0}(0)=f_{1}(0)=0$, then $\zeta_{f_{\sigma} \mid \mathbb{C}^{n}}(t)=(1-t) \times \zeta_{\left(f_{0} / f_{1}\right) \mid \mathbb{C}^{n}}(t)$, otherwise $\zeta_{f_{\sigma} \mid \mathrm{C}^{n}}(t)=$ $\zeta_{\left.\left(f_{0} / f_{1}\right)\right|_{c^{n}}}(t)$ (the zeta-function of the meromorphic function $f_{0} / f_{1}$ : [2]).

For $I \subset\{0,1, \ldots, n\}$ such that $0 \in I$, and for a covector $\alpha \in P^{I}$, assume that the face $\Gamma_{\alpha}^{I}(F)$ has dimension $l$, where $l=|I|-1>1$. Then $\Gamma_{\alpha}^{I}(F)$ is the convex hull of

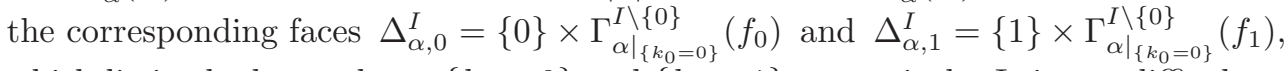
which lie in the hyperplanes $\left\{k_{0}=0\right\}$ and $\left\{k_{0}=1\right\}$ respectively. It is not difficult to show (see, e.g., [4, Lemma 1]) that $V_{l}\left(\Gamma_{\alpha}^{I}(F)\right)=V_{\alpha}^{I} / l$, where

$$
\begin{aligned}
V_{\alpha}^{I}= & V_{l-1}\left(\Delta_{\alpha, 0}^{I}, \ldots, \Delta_{\alpha, 0}^{I}\right)+V_{l-1}\left(\Delta_{\alpha, 0}^{I}, \ldots, \Delta_{\alpha, 0}^{I}, \Delta_{\alpha, 1}^{I}\right) \\
& +\ldots+V_{l-1}\left(\Delta_{\alpha, 0}^{I}, \Delta_{\alpha, 1}^{I}, \ldots, \Delta_{\alpha, 1}^{I}\right)+V_{l-1}\left(\Delta_{\alpha, 1}^{I}, \ldots, \Delta_{\alpha, 1}^{I}\right) .
\end{aligned}
$$

Here $V_{l-1}$ denotes the $(l-1)$-dimensional Minkowski's mixed volume: see, e.g., [8]. Moreover, $\alpha\left(\partial / \partial k_{0}\right)=\min \left(\left.\alpha\right|_{\Gamma^{I \backslash\{0\}}\left(f_{0}\right)}\right)-\min \left(\left.\alpha\right|_{\Gamma^{I \backslash\{0\}}\left(f_{1}\right)}\right)$, thus (2) coincides with the main result of [2].

\section{A'Campo type formula}

Proof of Theorem 2.2 uses an A'Campo type formula ([1]) written in terms of the integration with respect to the Euler characteristic ([3]).

For a constructible function $\Phi$ on a constructible set $Z$ with values in a (multiplicative) Abelian group $G$, its integral $\int_{Z} \Phi^{d \chi}$ with respect to the Euler characteristic $\chi$ is defined as $\prod_{g \in G} g^{\chi\left(\Phi^{-1}(g)\right)}$ (see [10]). Further we consider $G=\mathbb{C}(t)^{*}$ to be the multiplicative group of non-zero rational functions in the variable $t$.

Let $F$ be a germ of an analytic function on $\left(\mathbb{C}^{n+1}, 0\right)$ defined on a neighbourhood $U$ of the origin. Let $Y$ be a hypersurface in $\mathbb{C}^{n}$. Denote $S=\left(\mathbb{C}_{\sigma} \times Y\right) \cup\{\sigma=0\}$. Consider a resolution $\pi:(X, D) \rightarrow(U, 0)$ of the germ of the hypersurface $\{F=0\} \cup S$ at the origin, where $D=\pi^{-1}(0)$ is the exceptional divisor.

Theorem 3.1. Assume $\pi$ to be an isomorphism outside of $\pi^{-1}(U \cap S)$. Then

$$
\zeta_{f_{\sigma} \mid \mathbb{C}^{n} \backslash Y}(t)=\int_{D \cap W} \zeta_{\left.\Sigma\right|_{W \backslash Z}, x}(t)^{d \chi},
$$

where $W$ is the proper preimage of $\{F=0\}$ (i.e., the closure of $\pi^{-1}(V), V=$ $((\{F=0\} \cap U) \backslash S)), \Sigma=\sigma \circ \pi, Z=\pi^{-1}\left(\mathbb{C}_{\sigma} \times Y\right)$ and $\zeta_{\left.\Sigma\right|_{W \backslash Z}, x}(t)$ is the monodromy zeta-function of the germ of the function $\Sigma$ on the set $W \backslash Z$ at the point $x \in D \cap W$.

Proof. The map $\pi$ provides an isomorphism $W \backslash(Z \cup\{\Sigma=0\}) \rightarrow V$, which is also an isomorphism of fibrations provided by the maps $\Sigma$ and $\sigma$ over sufficiently small punctured neighbourhood of zero $\mathbb{D}_{\delta}^{*} \subset \mathbb{C}_{\sigma}$. Therefore the monodromy zeta-functions 
of this fibrations coincide, $\zeta_{\left.f_{\sigma}\right|_{\mathbb{C}^{n} \backslash Y}}(t)=\zeta_{\left.\Sigma\right|_{W \backslash Z}}(t)$ (the monodromy zeta-function of the "global" function $\Sigma$ on $W \backslash Z)$.

Applying the localization principle ([3]) to $\Sigma$ we obtain:

$$
\zeta_{\left.f_{\sigma}\right|_{\mathbb{C}^{n} \backslash Y}}(t)=\int_{W \cap\{\Sigma=0\}} \zeta_{\left.\Sigma\right|_{W \backslash Z}, x}(t)^{d \chi}
$$

The integration is multiplicative with respect to subdivision of its domain. One has $W \cap\{\Sigma=0\}=(D \cap W) \sqcup((W \cap\{\Sigma=0\}) \backslash D)$. Thus the right hand side of $(4)$ is the product

$$
\left[\int_{D \cap W} \zeta_{\left.\Sigma\right|_{W \backslash Z}, x}(t)^{d \chi}\right] \cdot\left[\int_{W \cap(\{\Sigma=0\} \backslash D)} \zeta_{\left.\Sigma\right|_{W \backslash Z}, x}(t)^{d \chi}\right] .
$$

The first factor coincide with the right hand side of (3); we prove that the second factor equals 1 .

For a point $x \in D$, its neighbourhood $U(x) \subset X$ with a coordinate system $u_{1}, u_{2}, \ldots, u_{n+1}$ is called convenient if each the of manifolds $D, Z$ can be defined on $U(x)$ by an equation of type $\mathbf{u}^{\mathbf{k}}=0$ and each of the functions $\Sigma, \tilde{F}=F \circ \pi$ has the form $a \mathbf{u}^{\mathbf{k}}$, where $a(0) \neq 0$. One can assume that $X$ is covered by a finite number of convenient neighbourhoods.

For an arbitrary convenient neighbourhood $U_{0}$, choose an order of coordinates $u_{i}$ on it such that $D=\left\{u_{1} u_{2} \cdots u_{l}=0\right\}$.

Proposition 3.2. The zeta-function $\zeta_{\left.\Sigma\right|_{W \backslash Z}, x}(t)$ at a point $x \in U_{0} \backslash D$ is well-defined by the coordinates $u_{l+1}, u_{l+2}, \ldots, u_{n+1}$ of $x$.

Proof. The germ of the manifold $Z$ at the point $x$ is defined by an equation

$$
u_{l+1}^{k_{1, l+1}} \cdots u_{n+1}^{k_{1, n+1}}=0 .
$$

In a neighbourhood of $x$ one has $\tilde{F}=a u_{l+1}^{k_{2, l+1}} \cdots u_{n+1}^{k_{2, n+1}}, \Sigma=b u_{l+1}^{k_{3, l+1}} \cdots u_{n+1}^{k_{3, n+1}}$, where $a(x) \neq 0, b(x) \neq 0, k_{1, j} \in\{0,1\} ; k_{2, j}, k_{3, j} \geq 0$. The zeta-function $\zeta_{\left.\Sigma\right|_{W} \backslash Z}, x(t)$ is well-defined by the numbers $k_{i, j}, i=1,2,3, j=l+1, \ldots, n+1$, which do not depend on $u_{1}, \ldots, u_{l}$.

For a rational function $Q(t)$, we define a set

$$
X_{Q}=\left\{x \in W \cap(\{\Sigma=0\} \backslash D) \mid \zeta_{\left.\Sigma\right|_{W \backslash Z}, x}(t)=Q(t)\right\} .
$$

It follows from the proposition above that for any convenient neighbourhood $U_{0}$ we have $\chi\left(U_{0} \cap X_{Q}\right)=0$. Thus for all $Q(t)$ we have $\chi\left(X_{Q}\right)=0$ and

$$
\int_{W \cap(\{\Sigma=0\} \backslash D)} \zeta_{\left.\Sigma\right|_{W \backslash Z}, x}(t)^{d \chi}=\prod_{Q} Q^{\chi\left(X_{Q}\right)}=1 .
$$




\section{Proof of Theorem 2.2}

Using the Newton diagram $\Gamma(F)$ of the germ $F$ on $\left(\mathbb{C}^{n+1}, 0\right)$, one can construct a unimodular simplicial subdivision $\Lambda$ of the set of covectors with non-negative coordinates $\left(\mathbb{R}^{n+1}\right)_{+}^{*}$ (see, e.g., [9]). Consider the toroidal modification map

$$
p:\left(X_{\Lambda}, D\right) \rightarrow\left(\mathbb{C}^{n+1}, 0\right),
$$

corresponding to $\Lambda$. Let $U \subset \mathbb{C}^{n+1}$ be a small enough ball with the centre at the origin, $X=p^{-1}(U), \pi=\left.p\right|_{X}$. Let $Y=\left\{z_{1} z_{2} \cdots z_{n}=0\right\} \subset \mathbb{C}_{\mathbf{z}}^{n}$. Then $S=$ $\left(Y \times \mathbb{C}_{\sigma}\right) \cup\{\sigma=0\}$ is the union of the coordinate hyperplanes of $\mathbb{C}^{n+1}$. Since $F$ is non-degenerate with respect to its Newton diagram $\Gamma(F), \pi$ is a resolution of the germ $S \cup\{F=0\}$ (see, e.g., [8]). Finally, $\pi$ is an isomorphism outside of $S$, so the resolution $(X, \pi)$ satisfies the assumptions of Theorem 3.1.

Compute the right hand side of (3). Let $x \in D \cap W$ be a point of the torus $T_{\lambda}$ of dimension $n-l+1$, corresponding to an $l$-dimensional cone $\lambda \in \Lambda$. Let $\lambda$ be generated by integer covectors $\alpha_{1}, \ldots, \alpha_{l}$ and let $\lambda$ lie on the border of a cone $\lambda^{\prime} \in \Lambda$ generated by $\alpha_{1}, \ldots, \alpha_{l}, \ldots \alpha_{n+1}$. Let $\left(u_{1}, \ldots, u_{n+1}\right)$ be the coordinate system corresponding to the set $\left(\alpha_{1}, \ldots, \alpha_{n+1}\right)$. There exists a coordinate system $\left(u_{1}, \ldots, u_{l}, w_{l+1}, \ldots, w_{n+1}\right)$ in a neighbourhood $U^{\prime}$ of the point $x$ such that $w_{i}(x)=0, i=l+1, \ldots, n+1$ and $\tilde{F}=F \circ \pi=a u_{1}^{k_{1,1}} u_{2}^{k_{1,2}} \cdots u_{l}^{k_{1, l}} \cdot w_{n+1}^{k_{1, n+1}}$ (where $\left.a(0) \neq 0\right)$. The zero level set $\{\Sigma=0\}$ is a normal crossing divisor contained in $\left\{u_{1} u_{2} \cdots u_{l}=0\right\}$. Therefore $\Sigma=\sigma \circ \pi=u_{1}^{k_{2,1}} u_{2}^{k_{2,2}} \cdots u_{l}^{k_{2, l}}$. One has: $W \cap U^{\prime}=\left\{w_{n+1}=0\right\}$ and

$$
(Z \cup\{\Sigma=0\}) \cap U^{\prime}=\left\{u_{1} u_{2} \cdots u_{l}=0\right\} .
$$

Thus $\zeta_{\left.\Sigma\right|_{W \backslash Z}, x}(t)=\zeta_{\left.g\right|_{\left\{u_{i} \neq 0, i<l\right\}}}(t)$, where $g$ is the germ of the following function of $n$ variables: $g\left(u_{1}, \ldots, u_{l}, w_{l+1}, \ldots, w_{n}\right)=u_{1}^{k_{2,1}} u_{2}^{k_{2,2}} \cdots u_{l}^{k_{2, l}}$.

Assume that one of the exponents $k_{2,1}, k_{2,2} \ldots, k_{2, l}$ (say, $k_{2,1}$ ) is equal to zero. Then $g$ does not depend on $u_{1}$. We may assume that the monodromy transformation of its Milnor fibre also does not depend on $u_{1}$. Denote $h=\left.g\right|_{\left\{u_{1}=0\right\}}$. The monodromy transformations of the fibre of $\left.g\right|_{\left\{u_{2} u_{3} \cdots u_{l} \neq 0\right\}}$ and one of $\left.h\right|_{\left\{u_{2} u_{3} \cdots u_{l} \neq 0\right\}}$ are homotopy equivalent, so $\zeta_{\left.g\right|_{\left\{u_{2} u_{3} \cdots u_{l} \neq 0\right\}}}(t)=\zeta_{\left.h\right|_{\left\{u_{2} u_{3} \cdots u_{l} \neq 0\right\}}}(t)$. On the other hand the multiplicative property of the zeta-function implies that

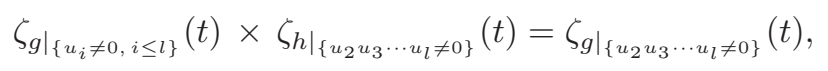

and thus $\zeta_{\left.g\right|_{\left\{u_{i} \neq 0, i \leq l\right\}}}(t)=1$.

Now assume that all the exponents $k_{2,1}, k_{2,2} \ldots, k_{2, l}$ are positive. Then the nonzero fibre of the function $g$ does not intersect $\left\{u_{1} u_{2} \ldots u_{l}=0\right\}$, so $\zeta_{\left.g\right|_{\left\{u_{i} \neq 0, i \leq l\right\}}}(t)=$ $\zeta_{g}(t)$. In the case $l>1$ one has $\zeta_{g}(t)=1$. In the case $l=1$ one has: $g=u_{1}^{k_{2,1}}$, $\zeta_{g}(t)=1-t^{k_{2,1}}$.

We see that the integrand in (3) differs from 1 only at points $x$ that lie in strata of dimension $n$. From here on $l=1$. If all the components of $\alpha=\alpha_{1}$ are positive, then 
$T_{\lambda} \subset D$. Otherwise, $T_{\lambda} \cap D=\emptyset$. From here on $\alpha \in P^{\{0,1, \ldots, n\}}$ (see the definitions before Theorem 2.2).

Using the coordinates $\left(u_{2}, \ldots, u_{n+1}\right)$ on the torus $T_{\lambda}=\left\{u_{1}=0\right\}$ we obtain: $T_{\lambda} \cap W=\left\{Q_{\alpha}=0\right\}$, where for the power series $F=\sum F_{\mathbf{k}} \sigma^{k_{0}} z_{1}^{k_{1}} \cdots z_{n}^{k_{n}}$ we denote $Q_{\alpha}=\sum_{\mathbf{k} \in \Gamma_{\alpha}^{\{0, \ldots, n\}}(F)} F_{\mathbf{k}} u_{2}^{\alpha_{2}(\mathbf{k})} u_{3}^{\alpha_{3}(\mathbf{k})} \cdots u_{n+1}^{\alpha_{n+1}(\mathbf{k})}$. So $T_{\lambda} \cap W$ is the zero level set of the Laurent polynomial $Q_{\alpha}$. Using results of $[5,6]$ we obtain:

$$
\chi\left(T_{\lambda} \cap W\right)=(-1)^{n-1} n ! V_{n}\left(\Delta\left(Q_{\alpha}\right)\right),
$$

where $\Delta(\cdot)$ denotes the Newton polyhedron. Since the polyhedra $\Delta\left(Q_{\alpha}\right)$ and $\Gamma_{\alpha}=$ $\Gamma_{\alpha}^{\{0,1, \ldots, n\}}(F)$ are isomorphic as subsets of integer lattices, their volumes are equal: $V_{n}\left(\Delta\left(Q_{\alpha}\right)\right)=V_{n}\left(\Gamma_{\alpha}\right)$. In a neighbourhood of a point $x \in T_{\lambda} \cap W$ one has $\Sigma=$ $a u_{1}^{\alpha\left(\partial / \partial k_{0}\right)}$, where $a(x) \neq 0$. Therefore $\zeta_{\left.\Sigma\right|_{W \backslash Z}, x}(t)=1-t^{\alpha\left(\partial / \partial k_{0}\right)}$. Thus one has:

$$
\int_{T_{\lambda} \cap W} \zeta_{\left.\Sigma\right|_{W \backslash Z}, x}(t)^{d \chi}=\left(1-t^{\alpha\left(\frac{\partial}{\partial k_{0}}\right)}\right)^{\chi\left(T_{\lambda} \cap W\right)}=\left(1-t^{\alpha\left(\frac{\partial}{\partial k_{0}}\right)}\right)^{(-1)^{n-1} n ! V_{n}\left(\Gamma_{\alpha}\right)} .
$$

Multiplying (5) for all strata $T_{\lambda} \subset D$ of dimension $n$ we get (1).

Acknowledgement. I am grateful to my advisor S. M. Gusein-Zade, who motivated me to make this study, for constant attention and support.

\section{References}

[1] N. A'Campo, La fonction zêta d'une monodromie, Comment. Math. Helv. 50 (1975), 233-248 (French).

[2] S. M. Guseřn-Zade, I. Luengo, and A. Melle-Herrnández, Zeta functions of germs of meromorphic functions, and the Newton diagram, Funktsional. Anal. i Prilozhen. 32 (1998), no. 2, 26-35, 95 (Russian, with Russian summary); English transl., Funct. Anal. Appl. 32 (1998), no. 2, 93-99.

[3] S. M. Guseln-Zade and D. Sirsma, Deformations of polynomials and their zeta functions, Sovrem. Mat. Prilozh. 33 (2005), 36-42 (Russian, with Russian summary); English transl., J. Math. Sci. (N. Y.) 144 (2007), no. 1, 3782-3788.

[4] G. G. Gusev, The Euler characteristic of the bifurcation set for a polynomial of degree two, Russ. Math. Surv. 63 (2008), no. 2, 363-365.

[5] A. G. Hovanskiı̌, Newton polyhedra, and toroidal varieties, Funkcional. Anal. i Priložen. 11 (1977), no. 4, 56-64, 96 (Russian).

[6] - Newton polyhedra, and the genus of complete intersections, Funktsional. Anal. i Prilozhen. 12 (1978), no. 1, 51-61 (Russian).

[7] Lê Dũng Tráng, Some remarks on relative monodromy, Real and complex singularities (Proc. Ninth Nordic Summer School/NAVF Sympos. Math., Oslo, 1976), Sijthoff and Noordhoff, Alphen aan den Rijn, 1977, pp. 397-403.

[8] M. Oka, Principal zeta-function of nondegenerate complete intersection singularity, J. Fac. Sci. Univ. Tokyo Sect. IA Math. 37 (1990), no. 1, 11-32. 
[9] A. N. Varchenko, Zeta-function of monodromy and Newton's diagram, Invent. Math. 37 (1976), no. $3,253-262$.

[10] O. Ya. Viro, Some integral calculus based on Euler characteristic, Topology and geometryRohlin Seminar, Lecture Notes in Math., vol. 1346, Springer, Berlin, 1988, pp. 127-138. 